\title{
Study of efficacy and complication of postpartum IUCD insertion at Govt. medical college, Bastar
}

\author{
Mamta Bansal*, Jyoti Lagoo, Khushbu Pujari
}

\begin{abstract}
Department of Obstetrics and Gynecology, Late B.R.K.M. Government Medical College, Jagdalpur, Chhattisgarh,
\end{abstract} India

Received: 13 October 2016

Accepted: 22 October 2016

*Correspondence:

Dr. Mamta Bansal,

E-mail: drmamtabansal1812@gmail.com

Copyright: (C) the author(s), publisher and licensee Medip Academy. This is an open-access article distributed under the terms of the Creative Commons Attribution Non-Commercial License, which permits unrestricted non-commercial use, distribution, and reproduction in any medium, provided the original work is properly cited.

\section{ABSTRACT}

Background: This study is aimed at determining the safety, efficacy and expulsion of PPIUCD. This study also aims to determine the rates of complications (bleeding, pelvic infection, lost strings, and displacement) following PPIUCD insertion among the women in one year period.

Methods: This study was conducted at department of obstetrics and gynecology, Late B.R.K.M Govt. medical college, Jagdalpur, Chattishgarh. Women admitted and delivered were counseled and those who fulfilled the medical eligibility criteria and had no contra indication for PPIUCD were provided the PPIUCD services.

Results: Total women 600, lost to follow-up 329, complications 162 (expulsion 14, bleeding 35, string problem 44), removal 102 .

Conclusions: The PPIUCD $(\mathrm{Cu} 380 \mathrm{~A})$ is demonstrably safe, effective, has high retention rate. The expulsion rate is not very high and it can be reduced with correct techniques, correct selection of clients, and correct time selection for the client.

Keywords: PPIUCD- Post partum intra uterine contraceptive device

\section{INTRODUCTION}

In India current method of family planning is (1) Family sterilization $34 \%$ (2) Male sterilization. $1 \%$ (3) pills $4 \%$ IUD $2 \%$ and condom $6 \%$ (4) any traditional method $7 \%$ (5) non user $46 \%$. The modern IUCD is highly effective, safe, long acting, coitus independent and rapidly reversible method of contraception with few side effects. It is most cost effective method of contraception today. Many women also find the IUCD to be very convenient because it requires little action once it is in place. ${ }^{1}$

The postpartum period is potentially an ideal time to begin contraception women are more strongly motivated to do so at this time, which also has the advantage of being convenient for both patients and healthcare providers. ${ }^{2}$ Postpartum period is a very vulnerable period both for women and infant. Initiation of contraception during this period is important to prevent unintended pregnancy and short birth intervals. It can avert more than $30 \%$ of maternal deaths and $10 \%$ of child mortality. Pregnancy occurring within six months of the last delivery holds a 7.5-fold increased risk for induced abortion and a 1.6-fold increased risk of stillbirth.

In India postpartum IUCD program is started by Family planning division of Ministry of Health and Family Welfare under Janani Shishu Suraksha Yojana, National Rural Health Mission in association with Bill and Melinda Gates Foundation, Norway.

India Partnership Initiative, JHPIEGO (India), USAID and Packard. The Government of India supplies CuT380A free of cost in all government institutes. 
This study was conducted to evaluate the safety and efficacy of Immediate Postpartum Intra Uterine Contraceptive Device (PPIUCD) insertion in women delivering vaginally or by caesarean section.

\section{Aims and objectives}

- To motivate the patient for Contraception

- To determine proportion of women accepting immediate PPIUCD insertion

- To study retention rate of postpartum IUCD

- To find out various complications of postpartum IUCD

- To study failure rate of Postpartum IUCD

- $\quad$ To study the expulsion rate of postpartum IUCD

\section{METHODS}

Design of the study was prospective.

\section{Period of study}

Cases were taken between January 2015 to June 2015 and they were following up till June 2016.

\section{Sample size}

Total number of women who opted PPIUCD during January 2015 to June 2016 i.e. 600.

\section{Study group}

Women's delivering in the hospital fulfilling inclusion criteria was included in the study after obtaining informed consent. The study protocol was approved by the ethics committee.

\section{Inclusion criteria}

All antenatal patients admitted for delivery to our hospital were counseled for PPIUCD. Consent was obtained from those, who opted for insertion; among those who fulfilled the following criteria were considered for inclusion.

- $\quad$ Age greater than or equal to 18 years

- Those who do not have any contraindications for IUD insertion.

\section{Exclusion criteria}

According to medical eligibility criteria for IUCD by WHO,

- $\quad$ Fever during labour and delivery (Temp >380c)

- Having active STD and other genital tract infection or high risk for STD

- Known to have ruptured membranes for $>18$ hrs prior to delivery
- Known uterine abnormalities ef. Bicornuate/septate uterus, uterine myomas

- Manual removal of the placenta

- Unresolved postpartum hemorrhage (PPH) requiring use of additional oxytocic agents in addition to AMTSL.

After selecting the women who fulfilled the eligibility criteria, detailed medical, obstetrical and gynecological history was taken and complete general physical as well as pelvic examination was done and the findings were recorded in the pre-designed Performa. The procedure was carefully explained to the women to make her as comfortable as possible.

In study group, Copper-T was inserted within 10 minutes of expulsion of placenta up to 48 hours postpartum in normal vaginal delivery, using Kelly's placental forceps and intracesarean insertion taking all aseptic precautions by standard technique. PPIUCD insertion is done on a delivery table, with adequate privacy for the woman and lighting for the service provider, with proper aseptic precautions. The following instruments and supplies are required for the postpartum insertion of IUCD. All the instruments are either sterilized or high level disinfected before use.

\section{RESULTS}

Table 1: Age distribution.

\begin{tabular}{|llll|}
\hline Age in years & Mean \pm SD & N & $\%$ \\
\hline $18-20$ & $19.67 \pm 0.6$ & 88 & 14.67 \\
\hline $21-25$ & $23.29 \pm 1.45$ & 236 & 39.33 \\
\hline $26-30$ & $28.12 \pm 1.6$ & 219 & 36.5 \\
\hline $31-35$ & $33.58 \pm 1.51$ & 50 & 8.33 \\
\hline$>35$ Years & $37.86 \pm 1.86$ & 7 & 1.17 \\
\hline Total & $25.55 \pm 4.32$ & 600 & 100 \\
\hline
\end{tabular}

Out of 600 cases, majority of patients belonged to 21-25 years age group (39.33\%). Mean age of women accepting the PPIUCD was $25.55 \pm 4.32$ year.

Majority of the patients opting for postpartum IUCD were primigravida and second gravid patients comprising of $68.17 \%$ i.e. 409 patients (Table 2).

Table 2: Parity.

\begin{tabular}{|c|c|c|}
\hline Parity & $\mathbf{N}$ & $\%$ \\
\hline Primi & 216 & 36 \\
\hline Two & 193 & 32.17 \\
\hline Three & 113 & 18.83 \\
\hline$\geq$ Four & 78 & 13 \\
\hline
\end{tabular}

Out of the 600 IUCD inserted, 66.5\% (399) were inserted after vaginal delivery and $33.5 \%$ (201) were kept intracesarean. In intracesarean majority were primigravida (Table 3 ). 
Table 3: Mode of delivery.

\begin{tabular}{|lll|}
\hline MOD & N & $\%$ \\
\hline Vaginal & 399 & 66.5 \\
\hline LSCS & 201 & 33.5 \\
\hline
\end{tabular}

Table 4: Timing of insertion.

\begin{tabular}{|lll|}
\hline Timing of Insertion & N & $\%$ \\
\hline POST Placental & 306 & 51 \\
\hline Postpartum $\leq 48$ hrs & 95 & 15.83 \\
\hline Intracesarean & 199 & 33.17 \\
\hline
\end{tabular}

Maximum number of IUCD i.e. 306 (51\%) were inserted post placental, followed by intracesarean 199 (33.17\%) and $95(15.83 \%)$ were inserted postpartum $(\leq 48 \mathrm{hrs})$.

Out of 600 , total follow up cases were 271 , in follow up cases following complication were seen.

Table 5: Complication.

\begin{tabular}{|lll|}
\hline Complication & N & $\%$ \\
\hline No complication & 109 & 40.22 \\
\hline Pain & 43 & 15.87 \\
\hline Bleeding & 35 & 12.92 \\
\hline Discharge & 26 & 9.59 \\
\hline Missing thread & 44 & 16.24 \\
\hline Expulsion & 14 & 5.17 \\
\hline Perforation & - & - \\
\hline
\end{tabular}

Reason for removal: During follow up 102 PPIUCD were removed.

Table 6: Reason for removal.

\begin{tabular}{|lll|}
\hline Reason for removal & N & $\%$ \\
\hline Pain & 8 & 7.84 \\
\hline Infection & 6 & 5.88 \\
\hline Bleeding & 18 & 17.65 \\
\hline Self-expulsion & 10 & 9.8 \\
\hline For conceiving & 13 & 12.75 \\
\hline For tubal ligation & 23 & 22.55 \\
\hline Vague causes & 24 & 23.53 \\
\hline
\end{tabular}

In the study it was observed that out of $102 \mathrm{CuT}$ removed, maximum number of $\mathrm{CuT}$ removed was due to vague causes i.e. $24(23.53 \%)$ and $17.65 \%$ removal were due to bleeding per vaginum. $36 \mathrm{CuT}$ were removed for nonmedical reasons i.e. for future conceiving and for tubal ligation.

\section{DISCUSSION}

During study period, 1959 women were delivered in our setup. All were counselled for IUCD insertion. Out of which 600 PPIUCD were inserted. i.e. $30.6 \%$ consented for insertion.
In present study the mean age of IUCD seekers was $25.55 \pm 4.32$ years which is similar to study by shah et al. ${ }^{3}$

In present study when compared with other parity groups, acceptance of the PPIUCD was higher among primiparous $(36 \%)$. Similar finding was reflected in the study done by safwat et al in Egypt, where $30 \%$ of primiparous accepted the use of PPIUCD compared to $15 \%$ of grand multiparous. ${ }^{4}$

In present study intracesarean insertion rate is $33.5 \%$ which is only $9.2 \%$ in shah et al. ${ }^{3}$

Out of 600 , total follow up cases were 271. Follow rate was $45.2 \%$ in one year. This finding is contrary to that found in Morrison et al i.e. $69 \%$; $66 \%$ in 6 month and one year and that found in Shah et al, i.e. $82.6 \%$ at 6 months, $50.8 \%$ at 1 year interval. ${ }^{3,5}$ These findings indicate a poor integration of vertical programs at all levels. "Insert and report and then forget" needs to be replaced by "Counsel and report, insert and report, and follow up and report" and of course provide service every time. This hospital is providing service to people around $200 \mathrm{~km}$ of area so the patient once taken services do not report back for follow up. They might take follow up services in nearby PHCs and CHCs.

Maximum number of IUCD i.e. 306 (51\%) were inserted post placental, followed by intracesarean 199 (33.17\%) and $95(15.83 \%)$ were inserted postpartum $(\leq 48 \mathrm{hrs})$.

In shah et al Maximum number of IUCD i.e. 128 (51.2\%) were inserted in immediate postpartum period, followed by postplacental $99(39.6 \%)$ and $23(9.2 \%)$ were inserted intra caesarean. ${ }^{3}$

$94(16.24 \%)$ among those inserted with PPIUCD had lost strings during first follow-up. Strings were found at cervical canal or needed ultrasound and confirmed that the IUCD were in situ. Missing thread rate was $8.69 \%$ seen in study by Mishra Sujnanendra. ${ }^{6}$

In the present study the incidence of abnormal bleeding was $12.92 \%$. Like other studies bleeding out numbers other complications. ${ }^{7}$

Expulsion rate in our study is $5.17 \%$. This was similar to a multicountry study done in Belgium, Chile and Phillippines which showed the rate of expulsion at 1 month ranging from 4.6 to $16 \%{ }^{8}$

In present study $9.59 \%$ complain of discharge per vaginum. The study by Morrison et al had lower rates of pelvic infection in Kenya (1\%) and in Mali (2\%). ${ }^{5}$

Husband and other family member's pressure for IUCD removal for vague reason was a significant reason $(23.53 \%)$ for removal next to bleeding and menstrual disturbance, these findings emphasize the important of involving the husband in prenatal counselling. 
It speaks of the importance of knowledge and motivation prior to insertion in continuing PPIUCD.

\section{CONCLUSION}

The insertion of immediate postpartum IUCD is safe and effective in the means of complications. Though it was a new concept for the population it was well accepted by community. The expulsion rates were though higher than the conventional

Interval method postpartum IUCD but it is also equally effective in regards of proper counselling, maintaining strict aseptic precautions and with proper insertion technique.

WE can conclude that Inserting CuT 380 A by 10 min after placental delivery is safe and effective, has high retention rate. The expulsion rate was not high, and further can be reduced with practice. With the high level of acceptance despite low levels of awareness, the government needs to develop strategies to increase public awareness of the PPIUCD through different media sources. We have to give more emphasis on follow up by counselling patients.

Cash incentives to the accepter, motivator and of course provider which has been started by our government would bring about a substantial progress in the PPIUCD use in developing countries like India.

Funding: No funding sources Conflict of interest: None declared

Ethical approval: The study was approved by the Institutional Ethics Committee

\section{REFERENCES}

1. Rutstein S. Further Evidence of the Effects of Preceding Birth Intervals on Neonatal, Infant, and Under-Five-Years Mortality and Nutritional Status in Developing Countries: Evidence from the Demographic and Health Surveys. DHS Working Papers No. 41. Macro International; 2008.

2. $\mathrm{Xu} J \mathrm{X}$, Reusche $\mathrm{C}$, Burdan $\mathrm{A}$. Immediate postplacental insertion of intrauterine device: A review of Chinese and world experiences. Adv Contracept. 1994;10:71-82.

3. Shah A. Nilesh. Evaluation of Safety Efficacy and Expulsion of PPIUCD, Indian $\mathrm{J}$ of Res. 2015;4(6):537-39.

4. Safwat. Acceptability of postpartum intrauterine contraceptive devices Med Prince Pract. 2003;12:170-5.

5. Charles Morrison. Clinical outcomes of two early postpartum IUD insertion programs in Africa, Contraception. 1996;53:17-2.

6. Mishra S. Evaluation of Safety, Efficacy, and Expulsion of Post-Placental and Intra-Cesarean Insertion of Intrauterine Contraceptive Devices (PPIUCD). The $\mathrm{J}$ of $\mathrm{Obs}$ and Gyne of India. 2014;64(5):337-43.

7. Celen S, Moroy P, Sucak A. Clinical outcomes of early post placental insertion of intrauterine contraceptive devices. Contraception. 2004;69:27982.

8. Blanchard H, Mac Kiag C. ACCESS-FP Program. 2006. Postpartum contraception: http://www.k4health.org/sites/default/files/postpartu mabortion_English.pdf

Cite this article as: Bansal M, Lagoo J, Pujari K. Study of efficacy and complication of postpartum IUCD insertion at Govt. medical college, Bastar. Int J Reprod Contracept Obstet Gynecol 2016;5:412831 . 